\title{
Teoría de la Acción Planeada y tasa de ejercicio percibida: un modelo predictivo en estudiantes adolescentes de educación física
}

\author{
Elisa Huéscar*, Jesús Rodríguez-Marín, Eduardo Cervelló y Juan A. Moreno-Murcia
}

Universidad Miguel Hernández de Elche

\begin{abstract}
Resumen: Este estudio se planteó como objetivo analizar algunos factores que influyen en la percepción de la tasa de ejercicio de estudiantes adolescentes españoles tomando como marco la teoría de la acción planeada (Ajzen, 1991). Participaron 698 estudiantes en clases de educación física que completaron el Cuestionario de la teoría de la conducta planeada (TCP) y el Cuestionario de Actividad Física Habitual. Los resultados del modelo de ecuaciones estructurales mostraron que la actitud, norma subjetiva y control de la teoría de la conducta planeada predecían positivamente la intención futura de practicar ejercicio físico, que, a su vez, predecía positivamente la tasa de ejercicio físico percibida por el adolescente. Los resultados de este estudio ofrecen apoyo a la teoría de la conducta planeada y destacan los beneficios de promover la práctica de ejercicio físico-deportivo a través del apoyo de los antecedentes motivacionales, actitud, norma subjetiva y control en la etapa de la adolescencia, dada la importancia de esta fase evolutiva en la instauración de hábitos saludables en los jóvenes.
\end{abstract}

Palabras clave: Teoría de la conducta planeada; motivación; intención; ejercicio físico; educación física.
Title: Theory of Planned Action and perceived rate of exercise: a predictive model for adolescent students in physical education.

Abstract: This study analyzes some factors that influence the perceived rate of exercise among Spanish adolescent students, using the theorical framework of the Theory of planned behavior (Ajzen, 1991). Participants were 698 physical education students who completed the questionnaires of the theory of planned behavior (TPB) and the Habitual Physical Activity Questionnaire. The results, via of structural equation modeling, showed that the attitudes, norms and control factors of the Theory of planned behavior predicts the future intention to practice physical exercise, and that intention predicts positively the adolescent's perceived rate of exercise. The results provide support for the Theory of planned behavior and highlight the benefits of promoting physical exercesi through support to the motivational antecedents of attitude, regulation and control in adolescence, given its importance in establishing healthy habits in young people.

Key words: Theory of planned behavior; motivation; intention; physical excercise; physical education.

\section{Introducción}

Pese a la abundante información proporcionada por muchos estudios sobre los múltiples beneficios para la salud de la práctica regular de ejercicio físico-deportivo (Castillo y Moncada, 2010; Molina-García, Castillo, y Pablos, 2007), aún parecen existir una serie de barreras en muchos de los jóvenes que les impiden comprometerse con éxito a cualquier programa de ejercicio físico. Por este motivo, es necesario producir trabajos centrados en los antecedentes psicosociales del ejercicio físico, para ofrecer una explicación ajustada a un fenómeno que podría empezar a suponer un problema de salud.

En este sentido, uno de los modelos que más investigación está generando en la actualidad, precisamente por su papel en la explicación de la toma de decisiones en contextos complejos, es la Teoría de la Conducta Planeada (TCP) (Ajzen, 1991, 2011; McEachan, Conner, Taylor, y Lawton, 2011), que es complementaria de la Teoría de la Acción Razonada (TRA) (Ajzen y Fishbein, 1980). Según sus postulados, la conducta de una persona, estaría determinada de forma inmediata por la intención que la persona tiene de realizar (o no realizar) esa conducta. A su vez, dicha intención de llevar a cabo un comportamiento es función de tres elementos fundamentales: en primer lugar, la actitud bacia la conducta como variable inicial, que viene determinada por las creencias de la persona sobre los resultados de la conducta que pretende, por la valoración de tales resultados, y por la

\footnotetext{
* Dirección para correspondencia [Correspondence address]:

Elisa Huéscar Hernández. Universidad Miguel Hernández de Elche. Departamento de Psicología de la Salud. Edificio Altamira. Avenida de la Universidad, s/n. 03202 Elche (Alicante, España).

E-mail: ehuescar@umh.es
}

experiencia, lo que se traduce en una forma aprendida de responder (favorable o desfavorable) a un evento de manera consistente (Ajzen, 1991). En segundo lugar, la norma subjetiva, que es el componente más social del modelo, e implica, a su vez, la creencia que la persona tiene de que existen presiones sociales que se ejercen sobre ella para que realice o no la acción en cuestión, y la motivación por complacer tales presiones, $\mathrm{y}$, en tercer lugar, la percepción de control comportamental que se tiene en la situación en la que se debe tomar la decisión y actuar.

Esa percepción es, también, función de las creencias que la persona tiene sobre sus propias capacidades para llevar a cabo un determinado comportamiento (González, Donolo, Rinaudo, y Paolini, 2011). Así pues, en el caso de la práctica de ejercicio físico-deportivo, según estos principios, pese a que una persona posea una actitud general positiva hacia el deporte porque considere que es probable que aumente su salud física y psicológica por el hecho de practicar ejercicio físico (expectativa, creencia sobre resultados) y le otorgue un valor positivo al hecho de que mejore su salud (valoración de resultados), la probabilidad de que lleve a cabo su propio plan de ejercicio físico va a depender también de su creencia de que su grupo de iguales le rechazaría si no practicara ningún tipo de ejercicio físico (expectativa, norma subjetiva), y cuánto le importa la opinión de los demás (motivación para cumplir con ellos), y, finalmente, de la creencia sobre cuál es su capacidad para llevar a cabo esa práctica deportiva (percepción de control). De esa manera, el comportamiento de práctica de ejercicio físico (conductual final), resultará de una decisión puramente subjetiva.

Azjen (1991) señala que la percepción de control de la conducta está determinada por variables externas, en este caso, por ejemplo, la oportunidad para la persona de practicar ejercicio físico-deportivo, y por variables de naturaleza inter- 
na, como podrían ser las percepciones de habilidad y eficacia para llevar a cabo esa acción. Así pues, de la suma de estos tres componentes, esto es, la actitud, la norma subjetiva y el control percibido de la conducta, resultaría la intención comportamental (Azjen, 1985), que sería el precursor más inmediato de la conducta. La intención hace referencia a la decisión de ejecutar o no una acción, y, dado que este aspecto es determinante para el comportamiento de las personas, dicha variable resulta clave en la predicción de numerosos comportamientos del ámbito social (Ajzen, 2011; Armitage, 2009; Pakpour et al., 2011).

En el ámbito del ejercicio físico, la TCP viene demostrando su utilidad en la comprensión y predicción de esta conducta ya desde la década de los noventa (e.g., Blue, 1995; Godin y Kok, 1996; Hagger, Chatzisarantis, y Biddle, 2002; Hausenblas, Carron, y Mack, 1997). Así pues, los resultados de estudios recientes señalan correlaciones positivas entre, por un lado, las variables actitud, norma subjetiva y control comportamental percibido, y por otro, la intención de practicar ejercicio físico (e.g., Downs y Hausenblas, 2005), destacando el papel de la intención como predictor dominante de la ejecución del comportamiento (Ries, 2010). No obstante, es importante puntualizar que, mientras que el modelo viene siendo útil en la predicción de la intención, respecto a la predicción del comportamiento en sí, la varianza explicada suele ser menor (Hagger et al., 2002). Esta distancia entre la intención y la conducta está siendo objeto de discusión en la literatura actual sobre el tema (McEachan et al., 2011; Ries y Sevillano, 2011), aludiendo en algunos trabajos a la existencia de barreras personales (e.g., la experiencia) o culturales (e.g., las costumbres) que podrían dificultar la conversión del "pretendo hacer" (intención) en "estoy haciendo" (conducta) (Fishbein y Ajzen, 2010).

Además, y a pesar de que existe una cantidad suficiente de investigación que justifica la estrecha relación entre las variables de la TCP y la disposición de la persona a comenzar a practicar ejercicio físico-deportivo, algunos trabajos (e.g., Armitage, 2005) están empezando a reclamar la necesidad de estudios que se centren no solo en el inicio de la práctica de ejercicio físico, sino también en el mantenimiento en el tiempo. En este sentido, en numerosos estudios se ha comprobado que la conducta pasada de la persona es un buen predictor de la conducta presente y futura (Arbinaga, García, Vázquez, Joaquín, y Pazos, 2011; Carpi, Zurriaga, González, Marzo, y Buuk, 2007), señalando incluso que esta variable podría tener más fuerza predictiva que la intención (Wong y Mullan, 2009). De esta forma, el hábito o conducta previa de la persona viene siendo incorporada a los trabajos de la TCP con la finalidad de conocer en qué medida la historia de la persona respecto a una conducta puede ayudar a predecir comportamientos posteriores. En el ámbito del ejercicio físico, son escasos los trabajos que relacionen la intención de seguir practicando ejercicio físico con la práctica realizada anteriormente, y los pocos que relacionan la TCP con la práctica deportiva excluyen en sus análisis variables relacionadas con los hábitos deportivos anteriores de la per- sona, centrándose ante todo en predecir la práctica futura de la persona. Así por ejemplo, Armitage (2005), en un estudio en el que intentaba relacionar la TCP con la práctica actual de ejercicio físico y su continuidad en los 12 meses posteriores, y en el que participaron 94 personas con edades comprendidas entre los 16 y 65 años de edad, encontró que tanto la actitud, norma subjetiva y control percibido de la TCP correlacionaban positivamente con la intención, aunque solamente la actitud y el control percibido correlacionaban positivamente con el comportamiento actual de la persona hacia la práctica, no encontrando relación correlacional entre la norma subjetiva y el comportamiento actual. En este mismo estudio, el control percibido de la conducta fue el predictor más fuerte tanto del inicio como del mantenimiento de la práctica físico-deportiva.

Por esta razón, y aunque el citado estudio se declaró pionero en su momento, son exiguos los estudios que sigan aclarando las relaciones entre todas estas variables del modelo clásico de la TCP de modo conjunto con la adición de una variable que refleje la ejecución de la conducta durante un tiempo considerado anteriormente. En su lugar, en la mayoría de ocasiones, se sigue apostando por la inclusión de variables emocionales al modelo clásico (e.g., Mohiyeddini, Pauli, y Bauer, 2009; Ries y Sevillano, 2011) provocando, en el ámbito deportivo, la necesidad de indagar en el papel que los hábitos conductuales respecto a la práctica físicodeportiva pudieran tener en el mantenimiento futuro de esa práctica por parte de la persona.

Por este motivo, el presente trabajo tiene como objetivo analizar algunos factores que influyen en la tasa de ejercicio percibida por estudiantes adolescentes españoles tomando como marco la teoría de la acción planeada (Ajzen, 1991). Para ello se plantea un modelo de ecuaciones estructurales donde se establece la hipótesis de que las variables, actitud, norma subjetiva y control percibido de la TCP correlacionarán positivamente entre sí; en segundo lugar, la hipótesis de que cada una de ellas predecirá positivamente la intención de practicar ejercicio físico-deportivo; y, por último, de manera novedosa en la investigación hasta el momento, la hipótesis de que la intención se relacionará positivamente tanto con los tres componentes de la TCP, como con la tasa de ejercicio físico percibida por la persona durante los últimos doce meses.

\section{Método}

\section{Participantes}

La muestra para este estudio estuvo compuesta por 698 estudiantes en clases de educación física (331 hombres y 367 mujeres), de edades comprendidas entre los 12 y 16 años $(M$ $=14.15, D T=1.44)$, pertenecientes a diez institutos públicos del territorio español. 


\section{Instrumentos}

\section{Cuestionario de la teoría de la conducta planeada}

Para la medida de las variables pertenecientes a la TCP (intención, creencias comportamentales, norma subjetiva y control comportamental percibido) se utilizó el "Cuestionario de la teoría de la conducta planeada" de Tirado, Neipp, Quiles y Rodríguez-Marín (2012), compuesto por un total de 20 ítems agrupados en cuatro factores: (1) norma subjetiva (4 ítems) (e.g. "la mayoría de las personas importantes para mí piensan que debería hacer ejercicio al menos 6 veces en las próximas dos semanas); (2) intención (4 ítems) (e.g. "he pensado hacer ejercicio al menos 6 veces en las próximas dos semanas); (3) control comportamental percibido (5 ítems) (e.g. "si yo quisiera podría hacer ejercicio al menos 6 veces en las próximas dos semanas); (4) actitud (7 ítems), empieza con una sentencia previa "Para mí hacer ejercicio al menos 6 veces en las próximas dos semanas sería...", planteándose en cada ítem un par de adjetivos opuestos (e.g. "muy malomuy bueno", "nada importante-muy importante", etc.), que se respondían con una escala Likert que oscilaba entre 1 para la actitud más negativa y 7 para la actitud más positiva. Para el resto de factores, todas las preguntas se respondían mediante una escala tipo Likert que oscilaba entre 1 (totalmente en desacuerdo) y 7 (totalmente de acuerdo), excepto un ítem del factor norma subjetiva que oscilaba entre 1 (nada de control) y 7 (mucho control). Los valores $\alpha$ obtenidos fueron .85 para el factor control comportamental percibido, .87 para el factor intención, .76 para el factor norma subjetiva y .86 para el factor actitud.

\section{Cuestionario de Actividad física habitual.}

Para medir la actividad física habitual de los participantes se utilizó la versión española (Sarria et al., 1987) del Cuestionario de Actividad Física Habitual de Baecke, Burema, y Frijters (1982). El cuestionario permite obtener tres puntuaciones de actividad física habitual referidas a los 12 últimos meses: actividades físicas ocupacionales, ejercicios físicos en el tiempo de ocio (PEL) y actividades físicas de ocio y locomoción (LLA). Como este estudio se realizó con estudiantes, solamente se utilizaron las puntuaciones del PEL y el LLA. La suma de las dos proporcionaba la puntuación total en actividad física habitual (TS). La puntuación del PEL se calculó por medio de cuatro preguntas. La primera de ellas hacía referencia al tipo de deporte o deportes practicados, la frecuencia semanal y los meses al año de práctica. La puntuación de esta primera pregunta se calculó aplicando la siguiente fórmula: Modalidad 1 (Intensidad x Tiempo x Proporción) + Modalidad 2 (Intensidad x Tiempo x Proporción). Para calcular dicha fórmula, según el deporte practicado, las horas semanales y los meses al año de práctica, se asignaron diferentes coeficientes (ver Ainsworth et al., 2000; Florindo y Latorre, 2003). Las otras tres preguntas valoraron el nivel de ejercicio físico en el tiempo de ocio (e.g. "durante el tiem- po de ocio practico deporte") a través de una escala Likert del 1 (nunca) al 5 (con mucha frecuencia). Para calcular la puntuación del PEL se reconvirtió la puntuación de la primera pregunta en valores del 1 al 5 y se calculará la media de las cuatro preguntas. Para calcular la puntuación del LLA se calculó la media de otras cuatro preguntas que valoraron el nivel de actividades físicas de ocio y locomoción (e.g. "durante el tiempo de ocio camino") por medio de una escala Likert del 1 (nunca) al 5 (con mucha frecuencia).

\section{Procedimiento}

Se llevó a cabo una selección de centros atendiendo a un muestreo aleatorio por conglomerados (Azorín, y Sánchez Crespo, 1986). Para la recogida de la información nos pusimos en contacto con diez Centros de Enseñanza Secundaria Obligatoria, informándoles de los objetivos del estudio y solicitando colaboración en el mismo. Una vez se recogió tanto la autorización del centro como el consentimiento informado de los padres de los estudiantes, en el que se informaba de la confidencialidad en el uso de los datos, se realizó la administración del cuestionario de las variables pertenecientes a la TCP (intención, creencias comportamentales, norma subjetiva y control comportamental percibido). Transcurridos doce meses se recogió la información de la actividad física habitual, en ambas ocasiones bajo la supervisión del investigador principal del estudio. El tiempo requerido para cumplimentar los cuestionarios fue de 25 minutos, aproximadamente.

\section{Análisis de los datos}

En primer lugar, se realizó un análisis descriptivo y de correlación de todas las variables del estudio. En segundo lugar, para comprobar la relación existente entre las variables propuestas, se empleó el método de dos pasos propuesto por Anderson y Gerbing (1988). En el primer paso, se testó la validez de constructo del modelo de medida a través de un análisis factorial confirmatorio (modelo de medición). Se dividieron los ítems que componían los factores latentes en dos grupos aleatorios, realizando dicho análisis basándonos en las 14 medidas observadas y los 7 constructos latentes que correlacionan libremente (Anderson y Gerbing, 1988). En el segundo paso, se realizó un modelo estructural con el que se analizaron las relaciones predictivas entre las variables analizadas. Todos los análisis se desarrollaron a través del paquete estadístico SPSS 19.0 y Amos 19.0.

\section{Resultados}

\section{Análisis descriptivo y de correlación de todas las va- riables}

Respecto a las variables de la TCP, la actitud fue la variable más valorada por los estudiantes obteniendo un valor medio de 5.63. La norma presentó una media de 5.53 y para 
el control se obtuvo una media de 4.27. La intención de ser físicamente activo presentó una valoración de 4.91 puntos sobre 7. Por su parte, la percepción de la tasa de ejercicio, obtuvo un valor medio de 5.08 (Tabla 1). El análisis de correlación reveló que la actitud, la norma, el control, la intención de ser físicamente activo y la percepción de la tasa de ejercicio correlacionaban positiva y significativamente entre sí.

Tabla 1. Media, Desviación Típica, Coeficiente Alfa y Correlación de las Variables.

\begin{tabular}{|c|c|c|c|c|c|c|c|c|}
\hline & $M$ & $\overline{D T}$ & $\alpha$ & Rango1 & 2 & 3 & 4 & 5 \\
\hline 1. Actitud & 5.63 & 1.10 & .88 & $1-7$ & $.37 * *$ & $.27 * *$ & $.40^{* *}$ & $.27 * *$ \\
\hline 2. Norma & 5.53 & 1.16 & .81 & $1-7-$ & - & $.44 * *$ & $.60^{* *}$ & $.33^{* *}$ \\
\hline 3. Control & 4.27 & 1.57 & .92 & $1-7-$ & - & - & $.64^{* *}$ & $.33^{* *}$ \\
\hline 4. Intención & 4.91 & 1.51 & .85 & $1-7-$ & - & - & - & $.37 * *$ \\
\hline $\begin{array}{l}\text { 5. Percepción de } \\
\text { tasa de ejercicio }\end{array}$ & 5.08 & 1.34 & .78 & $1-7-$ & - & - & - & - \\
\hline
\end{tabular}

\section{Modelo de medición}

Para poder realizar el análisis del modelo de medición y testar el modelo de ecuaciones estructurales (MEE), se redujo el número de variables latentes por factor, esto se aconseja especialmente cuando el tamaño de la muestra no es particularmente grande comparada con el número de variables del modelo (Marsh, Richards, Jonson, Roche, y Tremayne, 1994; Vallerand, 2001, 2007). Esta reducción puede ser lograda mediante la combinación de los ítems en pares. Así, la mitad de los primeros ítems de cada subescala fueron promediados para formar el primer bloque de ítems, y la segunda mitad de ítems fueron promediados para formar el segundo bloque de ítems, y así hasta el último. Marsh et al. (1994) propusieron el uso de los pares de ítems porque los resultados de éstos son más fiables, tienden a ser distribuidos de forma más normal, y porque se reduce a la mitad la ratio del número de variables medidas en el modelo y el número de los participantes del estudio.

Puesto que el coeficiente de Mardia fue elevado (26.1), en el análisis se utilizó el método de estimación de máxima verosimilitud junto al procedimiento de bootstrapping, que permitió asumir que los datos eran robustos ante la falta de normalidad (Byrne, 2001). De la misma forma, se consideraron una serie de coeficientes fit para evaluar la bondad de ajuste de los modelos de medición con los datos empíricos. Así, basándonos en las aportaciones de diferentes autores (Bentler, 1990; Bollen y Long, 1993; McDonald y Marsh, 1990), los índices fit ó índices de bondad de ajuste que se consideraron para evaluar la bondad del modelo de medición fueron: $\chi^{2}, \chi^{2} / g l$ RMSE $A$ (Root Mean Square Error of Aproximation), RMSR (Root Mean Square Residual) y los índices incrementales (CFI, IFI y TLI). Estos índices de bondad de ajuste son considerados aceptables cuando el $\chi^{2} / g l$ es inferior a 5 , los índices incrementales (CFI, IFI y TLI) son superiores a $.90 \mathrm{y}$ los índices de error (RMSEA y RMSR) son inferiores a .08 (Browne y Cudeck, 1993; Hu y
Bentler, 1999). Los índices obtenidos tras el análisis fueron: $\chi^{2}(30, N=698)=39.26, p<.000 ; \chi^{2} / g l=1.30 ; C F I=.99$; $\mathrm{NFI}=.98 ;$ TLI $=.99 ; \mathrm{RMSE} A=.02 ; \mathrm{RMSR}=.01$. También, se examinó la validez discriminante del modelo, respetando que la correlación entre las variables latentes, atenuadas por el error de medición (+/- 2 veces el error de medición), fuera inferior a 1.0. Por tanto, según las indicaciones anteriores, los resultados mostraron que el modelo de medición era adecuado.

\section{Modelo de ecuaciones estructurales}

Con el objeto de analizar las relaciones existentes entre las variables pertenecientes al modelo que se plantea (actitud, norma, control, intención y tasa de ejercicio físico percibida), se ha utilizado el Modelo de Ecuaciones Estructurales. Puesto que el coeficiente de Mardia fue elevado, en el análisis se utilizó el método de estimación de máxima verosimilitud junto al procedimiento de bootstrapping, que permitió asumir que los datos eran robustos ante la falta de normalidad (Byrne, 2001). El examen de bondad del modelo mostró los siguientes índices de ajuste: $\chi^{2}(21, N=698)=46.5, p<.000$, $\chi^{2} / g l=2.21, C F I=.99, \mathrm{NFI}=.98, T L I=.98, \operatorname{RMSE} A=$ $.04, \mathrm{RMSR}=.02$. Estos datos se ajustan a los parámetros establecidos, por lo que se puede aceptar como adecuado el modelo propuesto (Hu y Bentler, 1999). De igual modo, la contribución de cada uno de los factores a la predicción de otras variables se examinó a través de los pesos de regresión estandarizados. El valor " $\ell$ " asociado a cada peso se tomó como una medida de la contribución, de modo que valores superiores a 1.96 se consideran como significativos. En la Figura 1 se observa que la actitud, norma y control predijeron positivamente la intención de seguir practicando y ésta a su vez predijo la tasa de ejercicio físico percibida por el estudiante (18\% de varianza explicada).

\section{Discusión}

Tomando como marco la TCP (Azjen, 1991) este estudio tuvo como objetivo proporcionar un modelo integrador sobre las relaciones entre las variables actitud, norma subjetiva y percepción de control, la intención de practicar ejercicio físico y la tasa de ejercicio físico percibida por la persona durante los últimos doce meses. Los resultados del modelo de ecuaciones estructurales propuesto apoyaron las hipótesis planteadas, mostrando asociaciones positivas de la actitud, la norma subjetiva y el control percibido entre sí, y con la intención de practicar ejercicio físico-deportivo, la cual se relacionó también positivamente con la tasa de ejercicio físico practicado percibida.

Respecto a las relaciones positivas entre las variables antecedentes de la intención de la TCP (actitud, norma subjetiva y control comportamental percibido), los resultados encontrados coinciden con los postulados de dicha teoría, así como con la mayoría de estudios en esta línea (Armitage, 
2005; Downs y Hausenblas, 2005; Hagger et al., 2002). No obstante, en el trabajo de Ries y Sevillano (2011) la norma subjetiva de la muestra estudiada no correlacionaba, inesperablemente, con la actitud ni con el control conductual percibido.

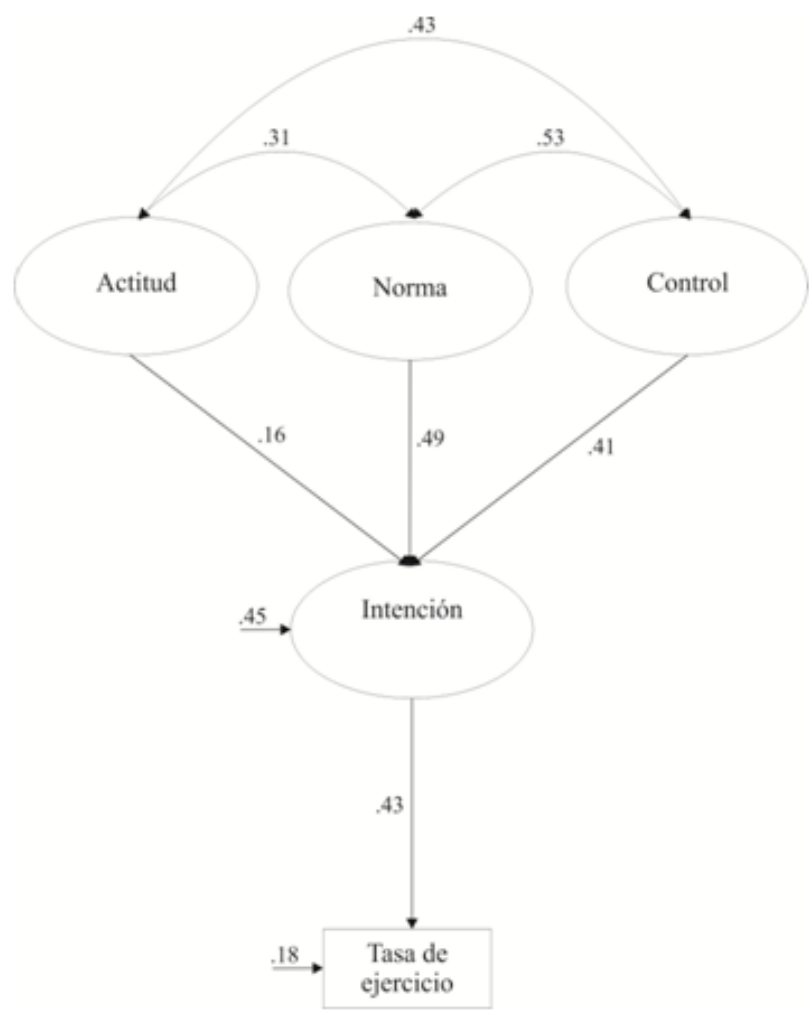

Figura 1. Modelo de ecuaciones estructurales que analiza las relaciones entre la actitud, norma, control, la intención y la percepción de la tasa de ejercicio.

Todos los parámetros son estandarizados y significativos en $p<.05$.

Los resultados revelan, además, que tanto la actitud, como la norma subjetiva y el control comportamental percibido se relacionaban positivamente con la intención de practicar ejercicio físico. En este sentido, ya en los inicios de esta teoría, Ajzen y Madden (1986) plantearon que estos tres componentes afectarían a la conducta mediante su efecto en las intenciones, de manera que, desde entonces, estos resultados vienen siendo corroborados en la mayor parte de estudios en este ámbito (Ajzen, 2011; Armitage, 2005; Hagger et al., 2002; McEachan et al., 2011). No obstante, en el estudio de Ries y Sevillano (2011) el control conductual percibido no aportaba nada significativo a la predicción de la intención, estando pues, determinada, únicamente por la actitud y la norma subjetiva (Ries y Sevillano, 2011). Aunque es cierto que, según Fishbein y Ajzen (2010) la importancia de los tres componentes de la TCP puede variar según la persona, en general, cabe esperar, que, aquellas personas que perciben un alto grado de control sobre su conducta muestren mayor práctica de actividad física. Así pues, la percepción de control podría influir directamente sobre la intención conductual, o indirectamente, modificando previamente la actitud y la norma subjetiva. A su vez, en la formación de la intención, la actitud se presenta como uno de los componentes con mayor poder, mientras que, en lo que a las conductas de salud se refiere, la norma subjetiva es el componente que, aún ejerciendo influencia sobre la intención, lo haría en menor medida que la actitud y el control percibido (Fuchs, 1996).

Con el objetivo de entender mejor estas relaciones de cara a su futura aplicabilidad en el contexto educativo y a partir del conocimiento del valor que tiene la intención como predictor de la conducta (Ajzen, y Fishbein, 2010; Balaguer, Castillo, Duda, Quested, y Morales, 2011) el modelo planteado en este estudio incluyó la hipótesis de que la intención se relacionaría positivamente con la tasa de ejercicio físico percibida por el estudiante durante los últimos doce meses. Los resultados obtenidos ofrecieron apoyo a esta hipótesis, indicándonos que las personas que muestran un mayor nivel de intención de seguir practicando ejercicio físico al terminar sus estudios, presentaban una mayor percepción de la tasa de ejercicio durante los doce meses anteriores. De esta forma, se entiende la intención como el potencial motivacional de la persona para hacer una actividad, la tasa de ejercicio percibida supondría conocer un paso más del proceso, materializando la intención en la conducta en sí, y dando pie a pensar que las personas que durante el último año han practicado ejercicio físico de una manera continuada lo seguirán haciendo en el futuro. Aunque en el ámbito de la educación física son escasos los estudios que analicen las relaciones entre estas variables, trabajos recientes relacionados con la TCP se han centrado en la importancia de la conducta pasada de la persona para la predicción de la conducta presente y futura (Sommer, 2011). Por ello, este estudio aporta resultados novedosos en esta línea de investigación aportando nuevas claves para la comprensión de las relaciones que contempla el paradigma de la TCP en el contexto de la promoción de la actividad física. No obstante, sería conveniente que otros estudios, además de seguir indagando en los factores psico-sociales que determinan tanto la motivación como sus consecuencias, focalizaran también en el papel de las dimensiones de la personalidad sobre la práctica físicodeportiva. Además, con el objetivo de alcanzar un análisis más preciso del comportamiento de la persona (Ajzen, Joyce, Sheikh, y Gilbert, 2011) sería relevante que futuros estudios reflejaran el análisis objetivo de la conducta, contrastando sus resultados con los obtenidos con las medidas de autoinforme empleadas en la evaluación de las variables de la TCP, así como complementar los resultados del presente estudio con otros de corte longitudinal donde se compruebe la percepción de la tasa de ejercicio en la adolescencia a edades posteriores.

En resumen, la aportación de un modelo teórico que relaciona los componentes de la TCP con la percepción de la tasa de ejercicio practicado en los últimos doce meses, entendido como el hábito de realizar ejercicio físico de manera continuada, ha mostrado que la cuantificación de la conducta de la persona en un intervalo de tiempo amplio, como puede ser un año, podría complementar las intervenciones 
dirigidas a desarrollar la promoción de actividad física en poblaciones sedentarias, ya que como se ha puesto de manifiesto en diferentes estudios, los hábitos pueden predecir la conducta futura directa o indirectamente a través de las actitudes o intenciones (Ajzen, 1991; Armitage, 2005; Sommer, 2011). Por tanto, consideramos que la inclusión de la medida de la práctica físico-deportiva de los últimos doce meses como vía de influencia sobre la conducta podría tener importantes implicaciones sobre el diseño de campañas de promoción de la salud dirigidas a la población joven con el

\section{Referencias}

Arbinaga, F., García, D., Vázquez, I., Joaquín, M., y Pazos, E. (2011). Actitudes hacia el ejercicio en estudiantes universitarios: relaciones con los hábitos alimenticios y la insatisfacción corporal. Revista Iberoamericana de Psicología del Ejercicio y el Deporte, 6(1), 97-112.

Armitage, C. J. (2009). Is there utility in the transtheoretical model? British Journal of Health Psychology, 14, 195-210.

Ainsworth, B. E., Haskell, W. L., Whitt, M. C., Irwin, M. L., Swartz, A. M., Strath, S. J., OBrien, W. L., Bassett, D. R. Jr., Schmitz, K. H., Emplaincourt, P. O., Jacobs, D. R. Jr., y Leon, A .S. (2000). Compendium of physical activities: an update of activity codes and MET intensities. Medicine and Science in Sports and Exercise 32, 498-516.

Ajzen, I. (1991). The theory of planned behavior. Organizational Behavior and Human Decision Processes, 50(2), 179-211.

Ajzen, I. (2011). The theory of planned behavior: Reflections and reflections. Psychology and Health, 26(9), 1113-1127.

Ajzen, I., y Fishbein, M. (1980). Understanding attitudes and predicting social behavior. Englewood Cliffs, N. J.: Prentices-All.

Ajzen, I., Joyce, N., Sheikh, S., y Gilbert Cote, N. (2011). Knowledge and the prediction of behavior: The role of information accuracy in the theory of planned behavior. Basic and Applied Social Psychology, 33(2), 101117.

Anderson, J. C., y Gerbin, D. W. (1988). Structural equation modeling in practice: a review y recommended two-step approach. Psychological Bulletin, 103, 411-423.

Azorín, F., y Sánchez-Crespo, J. L. (1986). Métodos y aplicaciones del muestreo. Madrid: Alianza Universidad.

Baecke, J. A., Burema, J., y Frijters, J. E. (1982) A short questionnaire for the measurement of habitual physical activity in epidemiological studies. American Journal of Clinical Nutrition, 36, 936-942.

Balaguer, I., Castillo, I., Duda, L. D., Quested, E., y Morales, V. (2011). Predictores socio-contextuales y motivacionales de la intención de continuar participando: Un análisis desde la SDT en danza. RICYDE. Revista Internacional de Ciencias del Deporte, 25(7), 305-319.

Bentler, P. M. (1990). Comparative fix indexes in structural models. Psychological Bulleting, 107, 238-246.

Blue, C. L. (1995). The predictive capacity of the theory of reasoned action and the theory of planned behavior in exercise research: an integrated literature review. Research in Nursing and Health, 18, 105-121.

Bollen, D. A., y Long, J. S. (1993). Testing structural equation models. Sage: Newbury Park, CA.

Browne, M. W., y Cudeck, R. (1993). Alternative ways of assessing model fit. En Bollen and Long (Eds.), Testing structura equation models (pp. 136-162). Newbury Park, CA: Sage.

Byrne, B. M. (2001). Structural equation modeling with Amos: Basic concepts, applications, and programming. Mahwah, NJ: Erlbaum.

Carpi, A., Zurriaga, R., González, P., Marzo, J. C., y Buuk, A. P. (2007). Incidencia de los hábitos de conducta en la prevención de la enfermedad cardiovascular. International Journal of Clinical and Health Psychology, 7, 5970 .

Castillo, I., y Moncada, J. (2010). El efecto de la frecuencia de participación en un programa de ejercicios contra resistencia sobre la estima y la satisfacción corporal de mujeres universitarias costarricenses. Revista Iberoamericana de Psicología del Ejercicio y el Deporte, 5(2), 195-212. objetivo de instaurar hábitos saludables que redunden en una mejor calidad de vida.

Agradecimientos.- La realización de este trabajo fue posible gracias al proyecto concedido por el Ministerio de Ciencia e Innovación "Desarrollo de estrategias sociales para el incremento de los niveles de actividad física y salud en adolescentes: una aproximación desde la teoría de la autodeterminación” (DEP2009-10610), en la convocatoria de Proyectos de Investigación Fundamental de la Dirección General de Investigación y Gestión del Plan Nacional de $\mathrm{I}+\mathrm{D}+\mathrm{i}$.

Downs, D. S., y Hausenblas, H. A. (2005). The theories of reasoned action and planned behavior applied to exercise: a meta-analytic update. Journal of Physical Activity and Health, 2, 76-97.

Fishbein, M., y Ajzen, I. (2010). Predicting and changing behavior: the reasoned action approach. New York: Psychology Press.

Florindo, A. A., y Latorre, M. R. D. O. (2003). Validation and reliability of the Baecke questionnaire for the evaluation of habitual physical activity in adult men. Revista Brasileira de Medicina do Esporte, 9, 129-135.

Fuchs, R. (1996). Causal models of physical exercise participation: testing predictive power of the constructe pressure to change. Journal of Applied Psychology, 26(21), 1931-1960.

Godin, G., y Kok, G. (1996). The theory of planned behavior: A review of its applications to health-related behaviors. American Journal of Health Promotion, 11, 87-97.

González, A., Donolo, D., Rinaudo, C., y Paolini, V. (2011). Relaciones entre motivación, emoción y rendimiento académico en universitarios. Estudios de Psicología, 32(2), 257-270.

Hagger, M. S., Chatzisarantis, N. L. D., y Biddle, S. J. H. (2002). A metaanalytic review of the theories of reasoned action and planned behavior in physical activity: Predictive validity and the contribution of additional variables. Journal of Sport and Exercise Psychology, 24, 3-32.

Hausenblas, H. A., Carron, A. V., y Mack, D. E. (1997). Application of the theories or reasoned action and planned behaviour to exercise behaviour: a meta-analysis. Journal of Sport and Exercise Psychology, 19, 36-51.

$\mathrm{Hu}, \mathrm{L} ., \mathrm{y}$ Bentler, P. M. (1999). Cutoff criteria for fit indexes in covariance structure analysis: Conventional criteria versus new alternatives. Structural Equation Modeling, 6, 1-55.

Marsh, H. W., Richard, G. E., Johnson, S., Roche, L., y Tremayne, P. (1994). Physical self-description questionnaire: Psychometric properties and a nultitrait-multimethod analysis of relations to existing instruments. Journal of Sport and Exercise Psychology, 16, 270-305.

McDonald, R. P., y Marsh, H. W. (1990). Choosing a multivariate model: Noncentrality and goodness of fit. Psychological Bulleting, 107, 247-255.

McEachan, R. R., Conner, M., Taylor, N., y Lawton, R. J. (2011). Prospective prediction of health-related behaviors with this Theory of Planned Behavior: A meta-analysis. Health Psychology Review, 5, 97-144.

Mohiyeddini, C., Pauli, R., y Bauer, S. (2009). The role of emotion in bridging the intention-behaviour gap: The case of sports participation. Psychology of Sport and Exercise, 10, 226-234.

Molina-García, J., Castillo, I., y Pablos, C. (2007). Bienestar psicológico y práctica deportiva en universitarios. Motricidad. European Journal of Human Movement, 18, 79-91.

Pakpour, A. H., Zeidi, I. M., Chatzisarantis, N., Moisted, S., Harrison, A. P., y Plotnikoff, R. C. (2011). Effects of action planning and coping planning within the theory of planned behavior: A physical activity study of patients undergoing haemodialysis. Psychology of Sport and Exercise, 12(6), 609-614.

Ries, F. (2010). Actividad fisico-deportiva en adolescentes de Sevilla y Luxemburgo: influencia de factores personales, parentales y situacionales percibidos sobre la intención y la práctica. Tesis doctoral. Universidad de Sevilla. [Online]. Recuperado de http://www.fondodigital.us.es. 
Ries, F., y Sevillano, J. M. (2011). Relación de las emociones y la actividad física dentro de la teoría de la conducta planificada. RICYDE. Revista Internacional de Ciencias del Deporte, 24, 158-173.

Sarria, A., Selles, H., Cañedo-Arguelles, L., Fleta, J., Blasco, M. J., y Bueno, M. (1987). A self-test for quantifying physical activity in adolescents. Nutrición Clínica y Dietética Hospitalaria 7, 56-61. (In Spanish: English abstract)

Sommer, L. (2011). The theory of planned behaviour and the impact of past behavior. International Business \& Economics Research Journal, 10(1), 91-110.

Tirado, S., Neipp, M. C., Quiles, M. J., y Rodríguez-Marín, J. (2012). Cuestionario de la teoría de la conducta planeada (TCP). Spanish Journal of Psychology, 15(2), 801-816.
Vallerand, R. J. (2001). A hierarchical model of intrinsic and extrinsic motivation in sport and exercise. En G. C. Roberts (Ed.), Advances in motivation in sport and exercise (pp. 263-319). Champaign, IL: Human Kinetics.

Vallerand, R. J. (2007). Intrinsic and extrinsic motivation in sport and physical activity. A review and a look at the future. En G. Tenenbaum y R. C. Eklund (Eds.), Handbook of sport psychology ( $3^{\mathrm{a}}$ ed., pp. 59-83). Nueva York: John Wiley.

Wong, C., y Mullan, B. (2009). Predicting breakfast consumption: An application of the theory of planned behaviour and the investigation of past behaviour and executive function. British Journal of Health Psychology, 14, 489-504.

(Artículo recibido: 5-11-2012; revisado: 18-3-2013; aceptado: 19-3-2013) 